\title{
A Macroscopic Model for High Intensity Radiofrequency Signal Detection in Swarm Robotics Systems
}

\author{
Fidel Aznar ${ }^{a *}$, M. José Pujol ${ }^{\mathrm{b}}$, \\ Mireia Sempere ${ }^{\mathrm{a}}$ and Ramón Rizo ${ }^{\mathrm{a} *}$ \\ ${ }^{a}$ Department of Computer Science and Artificial Intelligence at \\ University of Alicante, San Vicent del Raspeig, Alicante \\ (E-03080). Spain; \\ b Department of Applied Mathematics at University of Alicante, \\ San Vicent del Raspeig, Alicante (E-03080). Spain
}

\begin{abstract}
In recent years, there has been a growing interest in resource location in unknown environments for robotic systems, which are composed of multiple simple robots rather than one highly capable robot [12. This tradeoff reduces the design and hardware complexity of the robots and removes single point failures, but adds complexity in algorithm design. The challenge is to program a swarm of simple robots, with minimal intercommunication and individual capability, to perform a useful task as a group. This paper is focused on finding the highest intensity area of a radiofrequency $(\mathrm{RF})$ signal in urban environments. These signals are usually more intense near the city center and its proximity, since in these zones the risk of signal saturation is high. Radio frequency radiation (RFR) is boosted or blocked mainly depending on orography or building structures. RF providers need to supply enough coverage, setting up different antennas to be able to provide a minimum quality of service. We will define a micro/macroscopic mathematical model to efficiently study a swarm robotic system, predict their long-term behavior and gain insight into the system design. The macroscopic model will be obtained from Rate Equations, describing the dynamics of the swarm collective behavior. In our experimental section, the Campus of the University of Alicante will be used to simulate our model. Three RFR antennas will be taken into account, one inside our Campus and the other two in its perimeter. Several tests, that show the convergence of the swarm towards the RFR, will be
\end{abstract}

${ }^{* *}$ Corresponding author. Email:fidel@dccia.ua.es 
presented. In addition, the obtained RFR maps and the macroscopic behavior of the swarm will be discussed.

\section{Introduction}

Swarm robotics is an approach to solve problems inspired by the collective behaviors of social animals and it is focused on the interaction of multiple robots. Based on this metaphor of social insects, swarm robotics emphasizes aspects like decentralized control, limited communication between agents, local information, emergence of a global behavior and robustness [3]. Multi-swarm robotic systems differ from other multi-robotic systems because [11: 1) robots in a swarm are autonomous robots located in a certain environment, 2) the swarm has a large number of robots, 3) the swarm is composed of small groups of homogeneous robots, 4) robots will be relatively simple, and 5) the robots will have local sensors and their communication skills will be limited. These features ensure that the coordination between the robots will be distributed and that the system will be fault tolerant, since due to the redundancy of robots each of the agents in the system is not essential and can be replaced by another agent. Thus, the system is easily scalable, allowing more agents to be added or deleted according to task demands. Because of these features, it is difficult to develop an architecture that correctly models a swarm and, on the other hand, that coordinates the swarm to perform complex tasks.

In general, the robots behave like little insects, i.e., they don't have many skills individually, but when combined in large groups, they provide amazing results. Their underlying modular technology allows parallel tasks and has many applications, including: searching and rescuing people in natural disasters, replacing human workers in dangerous environments and exploring unknown or dangerous environments, among others. Swarm robotics is also used to identify the minimum requirements of agents that interact biologically, thus allowing the understanding of certain biological phenomena, such as the collective behavior of bacterial systems [13. In addition, robotic swarm models have been used for locating multiple sources of radiation resulting from nuclear accidents 6 .

\subsection{Presence of RF signals in an urban environment}

In urban environments there are many low- and high-frequency electromagnetic waves, where low-frequency signals correspond to transformers, transmission lines, electrical wiring, electrical appliances, etc., and high-frequency electromagnetic waves belong to mobile phone networks, wireless networks, radio and television, among others. Although radiofrequency (RF) signals operate in different wavelengths, the fact of using multiple wireless technologies at the same time, such as Bluetooth, GSM/GPRS or IEEE 802.11b, makes the environment to have interferences between the different signals, slowing down, as a result, the transmission speed. Moreover, there is a loss of performance when multiple users use the wireless network at the same time, because the medium access pro- 
tocol becomes inefficient. The quality of service for RF signals is influenced by different effects (such as geometric attenuation, atmospheric conditions, buildings, etc.); there are different kind of models to predict the attenuation [10], [1. In all cases, the theoretical models for chosing antennas (their locations and characteristics), must be validated by using field tests, in order to evaluate these undesired effects [2]. In general, we found that RF networks are exposed to a great source of noise produced by different elements, and these elements can have a significant impact on the interference level in the network 9]. Consequently, to keep the RF network performance, certain power levels are used to ensure signal links, but sometimes they generate more power than it is needed 5, 8. In this paper, we are interested in determining the quality of the mobile RF signal coverage in an urban area, identifying the areas where the greatest power of RF signal is received. From these results, we will determine if the intensity level is excessive or not in the identified areas.

\section{Design of the swarm}

An example of using extremely simple agents in swarm systems is described in the emergent game proposed in (http://icosystem.com/game.htm). In this game, an agent can develop three types of behavior (refugee or evasive, defender and attacker), from which complex behaviors emerge at the group level. These behaviors are discussed in [4], which shows that from these simple behaviors, three different behaviors of the swarm can be obtained: expansion, cycle or aggregation (in an unspecified place in the environment ${ }^{1}$ ). Obviously, this kind of behaviors does not allow exploration tasks or utilization of resources.

Inspired by these studies we propose to extend some of the behaviors outlined in 4. For our purposes we will use three behaviors: the aggressive agents, who pursue other agents; the recognition agents, that wander around the environment; and the elusive agents, whose main purpose is to escape from their attackers. As discussed in [4], if each agent pursues another agent, then the swarm tends to cluster at a specific point in the space. On the contrary, the behavior of elusive agents makes the swarm to expand infinitely.

Thus, we define an agent $r_{i} \in R$ as a vector $r_{i}=\left(s_{i}, p_{i}, a_{i}\right)$ containing states $s_{i} \in S$, sensors $p_{i} \in P$ and actuators $a_{i} \in A$. Each agent uses two actuators $a_{i}=\left(v_{\text {tras }}, v_{\text {rot }}\right)$ that allow the agent to set both the translational and rotational velocities. According to this, each agent will use 3 sensors $p_{i}=\left(m_{i}, c_{i}, t_{i}\right)$, where:

- $m_{i}$ : Sensor that allows getting the pair of an agent in the swarm. Each robot has a pair, so that $\forall m_{i} \in M, \forall m_{j} \in M, i \neq j, m_{i} \neq m_{j}$.

\footnotetext{
${ }^{1}$ Although the rules that define the operation of the agents are extremely simple, this not the case with the operation of the swarm. As discussed in [14], the design and evaluation of the behaviors that generate self-organization of swarm systems is a complex problem that depends on many factors, including the initial position of agents, the distribution of the environment extbackslashldots
} 
- $c_{i} \in[0,1]$ : Sensor that receives the RF signal intensity at the current position of the robot.

- $t_{i} \in[0, \infty[:$ robot's internal clock in order to know the current time.

On the other hand, an agent can be in one of three states $s_{i} \in\{$ expand, group, walk $\}$. expand refers to the elusive behavior of agents, where the agent $r_{i}$ has to calculate the repulsion vector with respect to the robot $m_{i}$, walk defines a random navigation behavior and group corresponds to the attraction behavior, where the attraction vector to $m_{j}$, should be calculated ${ }^{2}$ State changes are ruled by a probabilistic finite state machine (PFSM), defined by a quintuple $\left(\Sigma, S, s_{0}, \delta, F\right)$, where:

- $\Sigma$ is the input alphabet. In our case we will use only one symbol, $\Sigma=\left\{a_{\Phi}\right\}$ that will be emited every new cycle (so the transitions between states will be fired at each cycle).

- $S$ is a finite, non-empty set of states. More specifically $S=\{$ expand, group, walk $\}$.

- $s_{0}$ is an initial state, an element of $S$. Let us consider that $s_{0}=$ expand.

- $\delta$ is the state-transition function $\delta: S \times \Delta \rightarrow P(S)$. It is presented in matrix form in Figure 1

- $F$ is the set of final states. This is not used in our automata, so $F=\emptyset$.

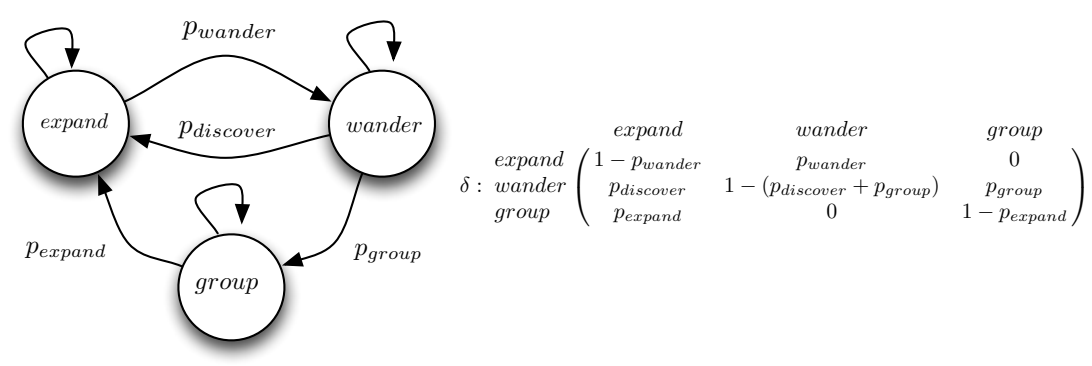

Figure 1: PFSM that rules the changes in the state of each one of the system agents.

Specifically, the probabilities $p_{\text {group }}, p_{\text {wander }}$ and $p_{\text {discover }}$ will be constant throughout the execution of the system. Their values depend on the investigator's specific interest in remaining more or less time in each of the states.

\footnotetext{
${ }^{2}$ The simplest way to calculate this vector requires to know the current position of the robot $\operatorname{pos}_{i}$ and the position of its pair $\operatorname{pos}_{m i}$ so that the repulsion vector can be defined as $v_{e}=\operatorname{pos}_{i}-\operatorname{pos}_{m_{i}}, \hat{v}_{\text {expand }}=\frac{\vec{v}_{e}}{\left\|\vec{v}_{e}\right\|}$ and the attraction vector is $\vec{v}_{g}=$ pos $_{m_{i}}-$ pos $_{i}, \hat{v}_{\text {group }}=$ $\frac{\vec{v}_{g}}{\left\|\vec{v}_{g}\right\|}$. Nevertheless, the attraction or repulsion vectors can be also calculated without knowing the current position of the robot, by using only indirect location methods.
} 
The agents start in the state expand and they will only be able to stay in one state at a time. From this state, each agent will calculate the new state at time $t$. In order to have enough time for the execution of behaviors, an agent can only change its state every $t_{c y c l e}$ seconds, and this time remains constant and

identical among all agents. This way, we define $\Phi=\frac{t}{t_{\text {cycle }}}$ as the number of cycles at time $t$.

Furthermore, $p_{\text {expand }}$ enables the swarm to expand once clustered, so that it keeps on exploring the environment from the area where it is at a certain time; this probability depends on the energy of each one of the agents. It would be interesting that when the system begins its operation, and thus the agents have more energy, the swarm begins to explore the environment, as well. As the energy of the agents decreases, we must ensure they group with the other members of the swarm. Thus, in Equation 1 we propose the following energy function, where $e_{i}$ is the initial energy of the agent $r_{i}, \Delta e$ is the decrease of the energy of the agent and $\Phi$ are the units of time elapsed.

$$
\left(\frac{e_{i}-\Delta e}{e_{i}}\right)^{\Phi}
$$

In addition, the system is designed so that when an agent is placed over a resource, its velocity will be slower so that the agent can inspect it and, if necessary, mark it for the rest of the swarm. Consequently, we propose to obtain a new vector $\vec{v}=\vec{v}_{b} \times c^{k}$, where $k$ is a modifier of the intensity of the attraction of the resource, $\vec{v}_{b}$ is the displacement vector obtained from the behavior to be developed by the robot at state $s_{i}$ and $c$ is the sensor that receives RF signal. From $\vec{v}$ both the rotational and translational velocities of the robot, $\left(v_{\text {tras }}, v_{\text {rot }}\right)$, will be obtained.

Moreover, if an agent perceives a collision with another agent, it will generate a random velocity vector $\vec{v}_{\text {rand }}$ until the danger of collision ends. This system prevents situations where a calculation needed for collision avoidance may require to fully know the environment nearby the agent, which is not always possible due to the limited perception these agents have.

Finally, we must point out that the initial position of the swarm is established by a matrix of up to 10 columns by an indeterminate number of rows (depending on the size of the swarm) such that pos init $\left(r_{i}\right)=\left\{i(\bmod 10),\left\lfloor\frac{i}{10}\right\rfloor\right\}$, so that the pair of each agent is defined as $m_{i}=r_{a}, a=i+1(\bmod |R|)$, where $|R|$ is the number of swarm robots.

\section{Macroscopic model}

Once the microscopic model of each of the agents is defined, let us determine now how the swarm will behave globally. Subsequently, this section will focus on providing a macroscopic model to predict what basic behavior will tend the swarm to. From the previously defined PFSM machine we can obtain the recurrence equations that rule the number of agents belonging to a state at any given time. 


$$
\left(\begin{array}{c}
\operatorname{expand}_{n+1} \\
\operatorname{wander}_{n+1} \\
\operatorname{group}_{n+1}
\end{array}\right)=\left(\begin{array}{ccc}
1-p_{\text {wander }} & p_{\text {discover }} & \left(\frac{e_{i}-\Delta e}{e_{i}}\right)^{\Phi} \\
p_{\text {wander }} & 1-\left(p_{\text {discover }}+p_{\text {group }}\right) & 0 \\
0 & p_{\text {group }} & 1-\left(\frac{e_{i}-\Delta e}{e_{i}}\right)^{\Phi}
\end{array}\right)\left(\begin{array}{c}
\text { expand }_{n} \\
\text { wander }_{n} \\
\text { group }_{n}
\end{array}\right)
$$

It is straightforward to show the stability of the system from the eigenvalues of the transition matrix. In Figure 7 the evolution of the system for an example of specific values is given. As shown, the system tends to stabilize with all of the robots being located in the aggregation state. This result is the expected one, since the energy of the agents decreases progressively and, as a consequence, the probability of going back to the expansion state decreases.

Paper [4] shows that the aggregation behavior for the agents that develop a clustering behavior is stabilized in a limited space of time as long as the connection between the agents constitute a connected graph. Therefore, as the proposed swarm tends to have all the agents located at the grouping behavior and it is connected, we can say that the swarm will tend to a cluster when the number of agents in state expand tends to 0 .

\section{Test environment for locating resources}

Given an area with radio frequency (RF) signal coverage, there will be usually an array of antennas to receive the signal, having different intensities and overlapping signal areas; there will be added elements, as well, either amplifying signals or attenuating them.

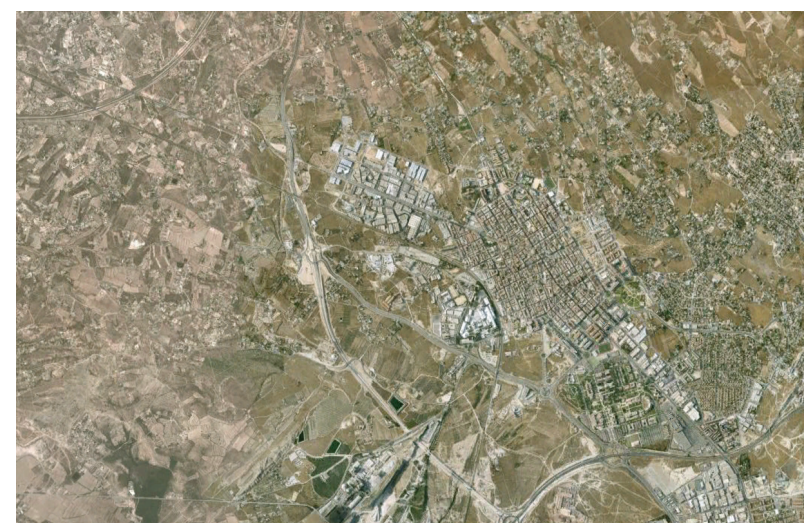

Figure 2: Urban area of the Campus of the University of Alicante and San Vicente surroundings $\left(38^{\circ} 23^{\prime} 13^{\prime \prime} \mathrm{N}, 0^{\circ} 30^{\prime} 45^{\prime} \mathrm{O}\right)$

Regarding the test area that has been used to validate our model, we must remark the following issues. First of all, the area is located in an area centered 
on the main Campus of the University of Alicante, as shown in Figure 2, Second of all, the RF signal coverage was generated using the program cloudrf (http:// cloudrf.com/), having three antennas located on the perimeter of the Campus. These antennas generate different signal intensities over the Campus, as shown in Figure 3, where clearer colors indicate greater signal intensities; these areas are also the ones with the highest amount of overlapping zones.

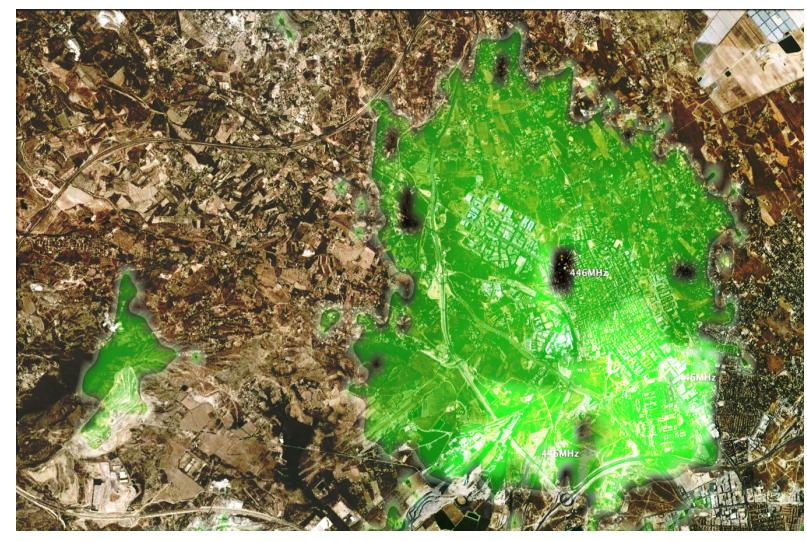

Figure 3: Urban area of the Campus of the University of Alicante with signal coverage areas

Additionally, in Figure 4 we present the perimeter of the global coverage area, where the service is provided by the RF network.

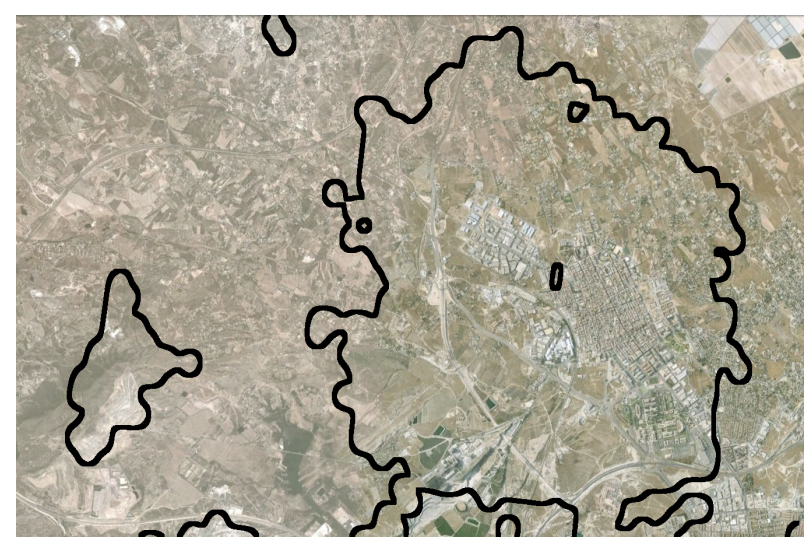

Figure 4: Global RF signal coverage area

As indicated in the swarm robot model, it is essential that robots will have a reduced cost for economy, robustness and viability reasons. In this case, we would use low cost quadricopters to fly over the exploration area at a preset altitude high enough to avoid colliding with the buildings. They will also have 
the necessary means for autonomous movement and a RF sensor to measure the signal strength. These devices may have production costs not exceeding $\$ 200$ per unit, which makes them viable for our proposed goal. Figure 5 shows a simulation of the flight of the swarm over a building in the Campus of the University of Alicante.

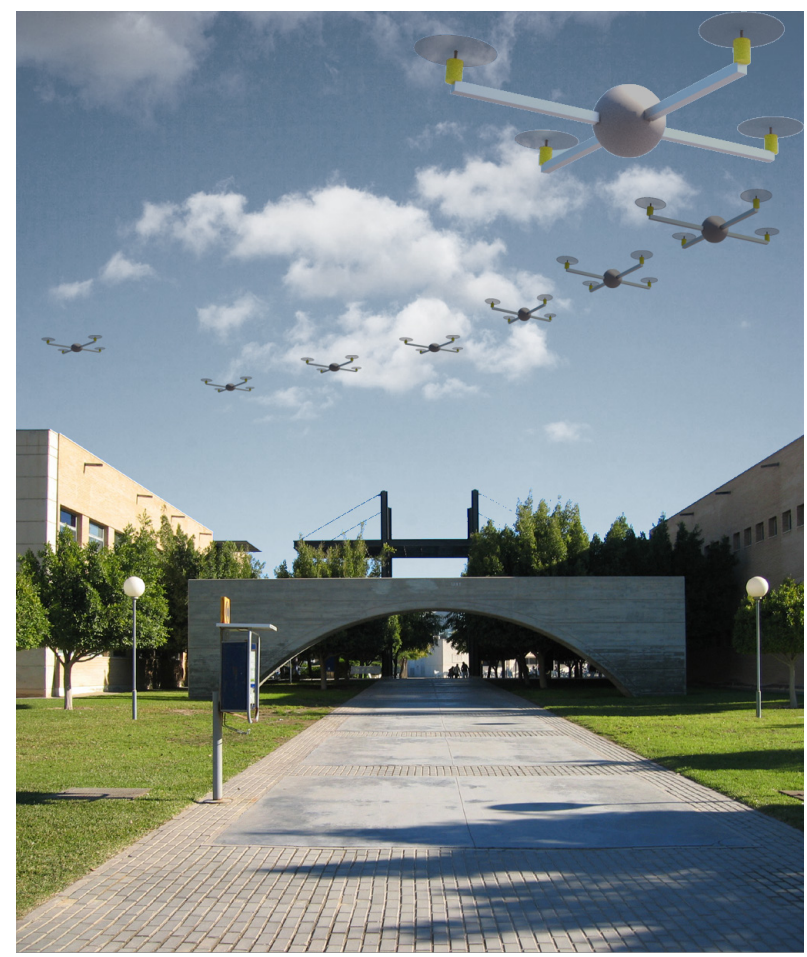

Figure 5: A simulation of the flight of the quadricopter swarm

\section{Experimentation}

For the simulation of the system, the powerful and versatile MASON simulator for multi-agent systems has been used [7]. Based on the MASON simulator, we have developed a continuous 2D environment, which contains discrete resource cells, and each cell has the same size as an agent (1x1m approx.). The resource map used by the swarm is presented in Figure 6 . The amount of resources of a cell (RF signal strength) is described from black (no resource) to white (maximum amount of resources). For each of the simulations there is an option to limit the maximum size of the environment (by default, it is assumed that the environment is bounded). In the simulation model, we consider different swarm sizes with $(10,50,100,500)$ individuals, whose objective is to locate the 
presence of signal and to indicate the areas of maximum coverage in a collective way, according to the behaviors defined by our model.

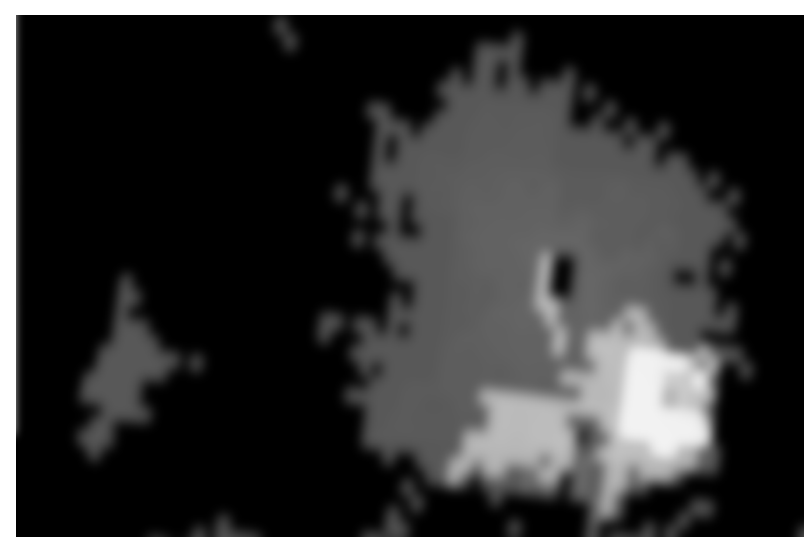

Figure 6: Resource Map (RF signal strength) used in our simulations. The RF signal strength is described from black (no detected signal) to white (highest signal strength). This map was generated with the program cloudrf. It uses the Irregular Terrain propagation Model (ITM), also known as the 'Longley Rice' model which was designed for planning UHF television broadcasting but has applications across a very wide proportion of the radio spectrum between 20 and 20,000 Megahertz

There are two purposes in doing this. On the one hand, we would like to show that the macroscopic model proposed above is correct and it corresponds to the behavior of the swarm. On the other hand, we want to verify that the swarm is able to locate the area with highest RF intensity level in the environment.

We have obtained the PFSM probabilities with metaheuristics using swarm convergence as measure quality. After training the system with several theoretical RF maps, generated for different size environments and number of antennas, we obtained the probability values for the PFSM machine that maximizes the convergence of the swarm. The following probabilities $\left(p_{\text {wander }}, p_{\text {discover }}, p_{\text {group }}\right)=$ $(0.274,0.154,0.222)$ will be used for the tests. In addition, each agent will make use of the following energy values: $\left(e_{i}, \Delta e\right)=(500,35)$.

We performed 50 independent executions of the system for each swarm size, and compared the theoretical model with the actual model. The results are presented in Figure 7 and Figure 9. From these results, the theoretical model accurately predicts the state of the agents at a given time. On the other hand, the convergence of the system to the area of greatest intensity has also been verified. In Figure 8 the convergence of the Euclidean distance of the centroid of the swarm with respect to the area of maximum RF signal for a number of iterations and a given swarm size is considered. Thus, the convergence of the system for different sizes of the swarm has also been shown. Although for small swarms deviation is larger (and therefore the movements of the swarm for the 


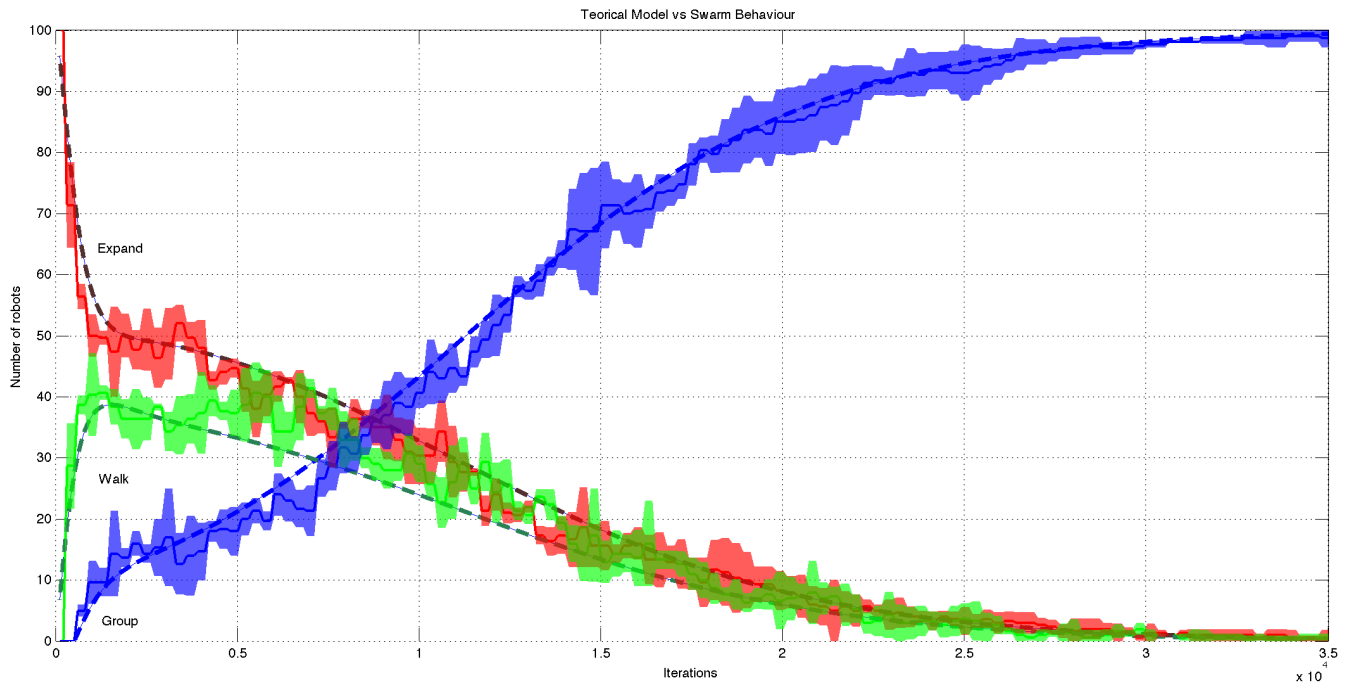

Figure 7: This figure shows the number of agents in each of the three possible states (wander, discover and group) for both the theoretical model (dashed lines) and the experimental data (red, green and blue areas). We used a swarm of 100 robots, with the following values of probability and energy: $\left(p_{\text {wander }}, p_{\text {discover }}, p_{\text {group }}, e_{i}, \Delta e\right)=(0.274,0.154,0.222,500,35)$. Initially, all the robots are in the expansion state. The experimental data represent the mean and standard deviation of the most representative 5 independent executions 
environment are less directed), the area of greatest RF intensity is achieved after virtually using the same number of iterations as in larger swarms.

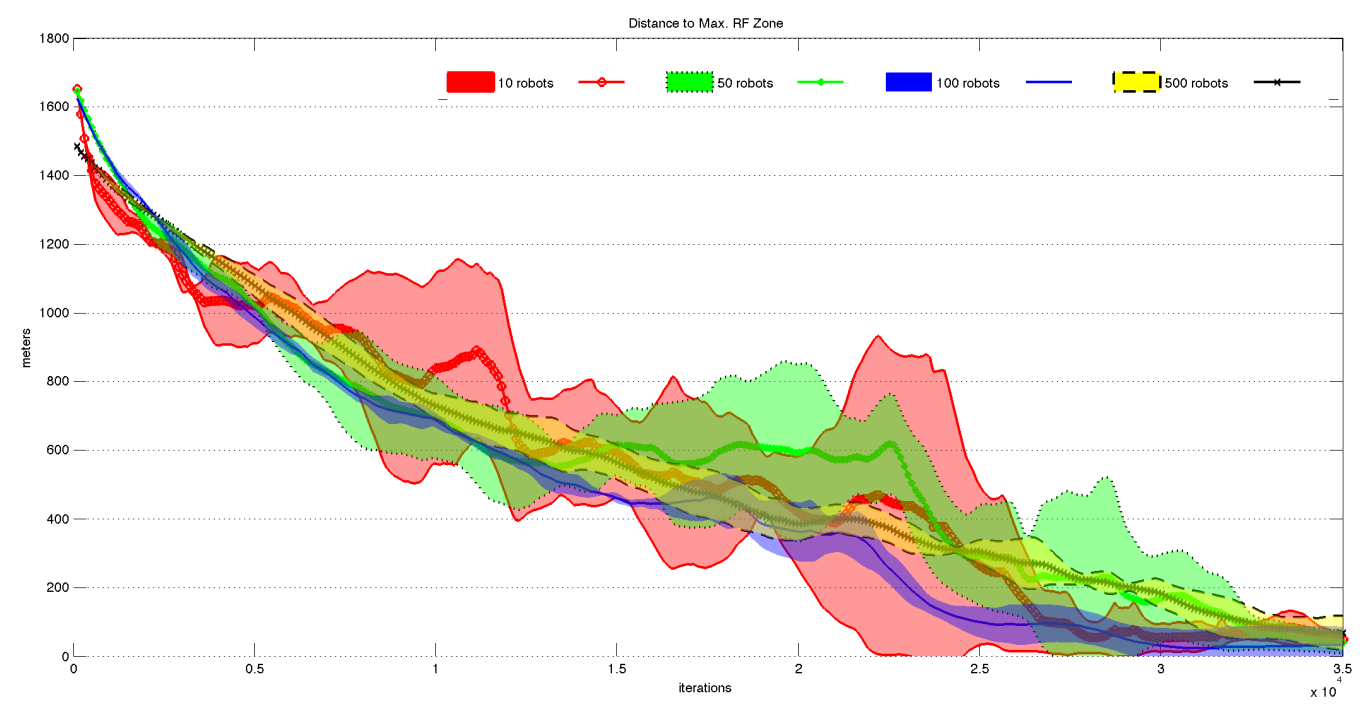

Figure 8: Convergence of the swarm over the area of highest RF intensity. Swarms of $(10,50,100,500)$ individuals have been used. This graph represents the Euclidean distance of the swarm to the area of maximum RF signal. Specifically, for each swarm size the most representative 5 independent tests were presented, showing the mean and standard deviation in all the cases

\section{Conclusions and future works}

As shown in Figure 9, once the process of locating ends, the individuals of the swarm are located on sites that have the highest RF intensities. This allows engineers to determine how to distribute the signal more efficiently by means of two alternatives: firstly, by modifying the transmission power of the antennas and, secondly, by relocating some of them. In any case, the proposed method verifies that the theoretical model of RF signals in an urban area can be validated using the swarm model proposed in this paper. We therefore consider that the data provided demonstrated empirically that the swarm will always aggregate in one of the highest RF signal locations. This application of swarm systems can establish new ways to improve signal coverage and to reduce the signal strength in areas with enough intensity levels.

We are currently developing the prototype of drones to be used in the swarm. We are interested in testing the models here presented in real environments, as well as addressing other application areas: surveillance, rescue, identifying agents or mapping inaccessible environments such as caves or grottoes. Another 


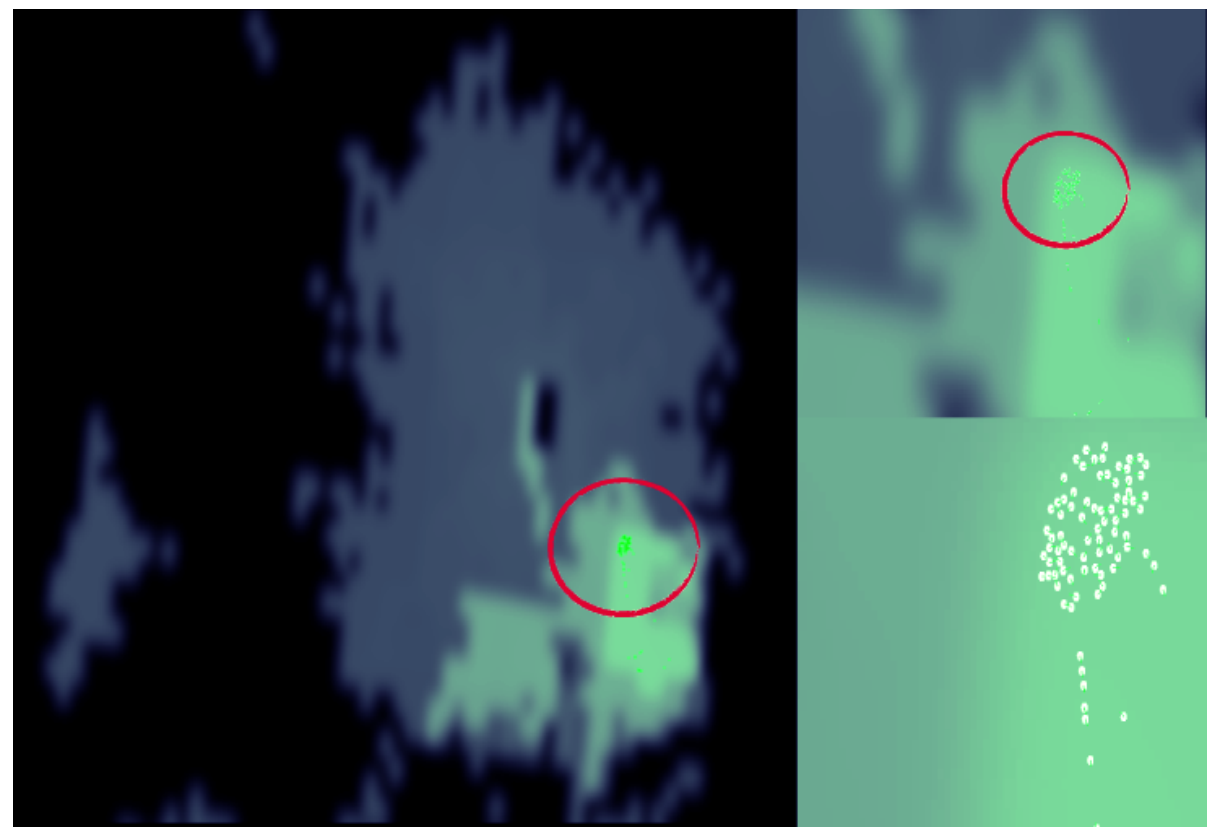

Figure 9: Convergence of the swarm over the area of highest RF intensity for a swarm of 100 individuals. The convergence is obtained at iteration 25,000. We present two extensions to the areas of greatest density of robots 
future research line is to determine analytically the convergence point of the swarm and to analyze the performance of our model with different sizes of swarms formed by heterogeneous agents, as well as to identify new behaviors at both microscopic, and macroscopic and social levels.

\section{Acknowledgement}

This work has been supported by the Spanish Ministerio de Ciencia e Innovación, project TIN2009-10581.

\section{References}

[1] A. Akbulut, H.G. Ilk, and F. Ari. Design, availability and reliability analysis on an experimental outdoor fso/rf communication system. In Transparent Optical Networks, 2005, Proceedings of 2005 7th International Conference, volume 1, pages 403 - 406 Vol. 1, july 2005.

[2] M. Chabane M. Alnaboulsi H. Sizun Bouchet, T. Marquis. Fso and quality of service software prediction. Proc. SPIE 5892, 589204, 2005.

[3] Marco Dorigo, Elio Tuci, Roderich Groß, Vito Trianni, Thomas Halva Labella, Thomas Halva, Shervin Nouyan, Christos Ampatzis, Jean louis Deneubourg, Gianluca Baldassarre, Francesco, Stefano Nolfi, Francesco Mondada, Dario Floreano, and Luca Maria Gambardella. The swarm-bot project. In Kunstliche Intelligenz, pages 31-44. Springer Verlag, 2005.

[4] Ian A. Gravagne and Robert J. Marks. Emergent behaviors of protector, refugee, and aggressor swarms. IEEE Transactions on Systems, Man, and Cybernetics, Part B, 37(2):471-476, 2007.

[5] Ramakrishna Gummadi, David Wetherall, Ben Greenstein, and Srinivasan Seshan. Understanding and mitigating the impact of $\mathrm{rf}$ interference on 802.11 networks. In Proceedings of the 2007 conference on Applications, technologies, architectures, and protocols for computer communications, SIGCOMM '07, pages 385-396, New York, NY, USA, 2007. ACM.

[6] K.N. Krishnanand, P. Amruth, M.H. Guruprasad, S.V. Bidargaddi, and D. Ghose. Glowworm-inspired robot swarm for simultaneous taxis towards multiple radiation sources. In Robotics and Automation, 2006. ICRA 2006. Proceedings 2006 IEEE International Conference on, pages 958 -963, may 2006.

[7] Sean Luke, Claudio Cioffi-Revilla, Liviu Panait, and Keith Sullivan. Mason: A new multi-agent simulation toolkit. Proceedings of the 2004 SwarmFest Workshop, 2004. 
[8] Henrik Lundgren, Anand Prabhu Subramanian, Theodoros Salonidis, Marianna Carrera, and Pascal Le Guyadec. Interference mitigation in wifi networks using multi-sector antennas. In Proceedings of the 4 th ACM international workshop on Experimental evaluation and characterization, WINTECH '09, pages 87-88, New York, NY, USA, 2009. ACM.

[9] Aniket Mahanti, Niklas Carlsson, Carey Williamson, and Martin Arlitt. Ambient interference effects in wi-fi networks, 2010.

[10] F. Nadeem, E. Leitgeb, M.S. Awan, and G. Kandus. Comparison of different models for prediction of attenuation from visibility data. In Satellite and Space Communications, 2009. IWSSC 2009. International Workshop on, pages $269-273$, sept. 2009.

[11] Erol Şahin. Swarm robotics: From sources of inspiration to domains of application, 2005.

[12] Mireia Sempere, Fidel Aznar, Mar Pujol, and Ramon Rizo. On cooperative swarm foraging for simple, non explicitly connected, agents, 2010.

[13] Adi Shklarsh, Gil Ariel, Elad Schneidman, and Eshel Ben-Jacob. Smart swarms of bacteria-inspired agents with performance adaptable interactions. PLoS Comput Biol, 7(9):e1002177, 092011.

[14] Onur Soysal and Erol Sahin. A macroscopic model for self-organized aggregation in swarm robotic systems, 2007. 